\title{
DISSIPATION AND THE FORMATION OF GALAXIES
}

\author{
R.G. Carlberg \\ York University and CITA, Toronto \\ and \\ Johns Hopkins University, Baltimore.
}

\begin{abstract}
The evidence for dissipation in elliptical galaxies indicates neither the epoch of formation nor the rate of radiation. The hypotheses for the formation of ellipticals include mergers of pre-existing, mostly stellar, disk galaxies; accumulation of gassy fragments that subsequently turn into stars; and the dynamical collapse of a distinct protogalactic gas cloud with simultaneous star formation. Mergers of purely stellar disks seem unlikely, because the phase space density of disks is everywhere far below that of the cores of normal ellipticals. Allowing a few percent of the mass of the galaxy to dissipate into the core and turn into stars could remove this difficulty. In the Hubble sequence of galaxies, ellipticals are characterized by their low angular momentum content. As a start to understanding the general problem for galaxy formation and angular momentum acquisition in the presence of dissipation, a cosmological $\mathrm{N}$-body experiment containing both a dominant collisionless component and an isothermal gas is described. The collisionless component clusters in the usual hierarchical manner appropriate to the spectrum of fluctuations. In contrast, the gas fragments only when the Jeans mass drops below the turnaround mass. The fragments subsequently shrink, becoming distinct entities with relatively low chances of being quickly incorporated in a larger unit. Gravitational torques transfer angular momentum outward in the dissipating gas, placing most of the gas angular momentum at large radii in the protogalaxy. The distant, high angular momentum gas has a relatively long infall time onto the galaxy. The gas may continue to rain down for some time if the galaxy remains undisturbed, or, the growth of clustering may strip the gas off, leaving a low angular momentum system.
\end{abstract}

\section{THE EVIDENCE FOR DISSIPATION}

The case for dissipation being a dominant process in the formation of elliptical galaxies largely rests on circumstantial evidence. Galaxies are an apparent break in the clustering hierarchy, since their internal luminosity densities are 1000 times higher than the extrapolation of the correlation function to the sizes of galaxies would predict (Efstathiou, Fall and Hogan 1979). Either galaxies suffered a radial contraction of a factor of 10 , or galaxy formation and galaxy clustering arise independently. Whether dissipation was an intrinsic part of the formation of ellipticals is less clear. If dissipation and star formation take place in a common process they leave behind radial chemical abundance gradients, such as those measured (in this volume see papers by Efstathiou, and Davies and Sadler). Metallicity gradients 
do exist in elllipticals, but in contrast to the manifestly dissipated disk galaxies, ellipticals do not appear to have distinct chemical-kinematic stellar populations. In any case, the observation that large ellipticals are not rotationally flattened (Illingworth 1977, Davies, et al. 1983) means that they cannot be formed in dynamical equilibrium from a gaseous protogalaxy.

A case for radiative cooling is made using the average mass densities and virial temperatures of elliptical galaxies, that together imply gas cooling times less than a free fall time (Binney 1977, Rees and Ostriker 1977, Silk 1977). A possible caveat is that the cooling is calculated under the optically thin assumption, whereas the high average surface density of an elliptical implies an optical depth greater than unity to electron scattering alone (Fish 1964).

\section{DISK GALAXY MERGERS AND DISSIPATION}

Collisions between disk galaxies quite plausibly produce "star piles" having characteristics similar to ellipticals (Toomre 1977). An extreme version of this hypothesis is open to observational test. The phase space density cannot increase unless there is dissipation or two body relaxation. Comparison of the phase space densities in disk galaxies to those in ellipticals should find that the ellipticals have lower values.

The phase space density is estimated as the product of the volume density and a gaussian velocity distribution. The peak phase space density in the core of an elliptical is then,

$$
\begin{aligned}
f_{c} & =\frac{M}{L} \frac{\rho_{L}}{\left(2 \pi \sigma_{c}^{2}\right)^{3 / 2}} \\
& =\frac{9 \sigma_{c}^{2}}{2 \pi G I_{c} r_{c}} \frac{I_{c}}{2 r_{c}} \frac{1}{\left(2 \pi \sigma_{c}^{2}\right)^{3 / 2}} \\
& =\frac{9}{2(2 \pi)^{5 / 2} G} \frac{1}{\sigma_{c} r_{c}^{2}}
\end{aligned}
$$

where $\rho_{L}$ is the central luminosity density, $I_{c}$ is the central surface brightness, $r_{c}$ is the radius at which the brightness drops to half of $I_{c}$, and $\sigma_{c}$ is the central velocity dispersion. The constant is $10.5 \mathrm{M}_{\odot} \mathrm{pc}^{-3}\left(\mathrm{kms}^{-1}\right)^{-3}$ when $\sigma_{c}$ is measured in $\mathrm{km} \mathrm{s}^{-1}$ and $r_{c}$ in parsecs.

The central phase space densities of disks are calculated in a similar manner. Using the locally isothermal, constant scale height, model advocated by van der Kruit and Searle (1982) gives,

$$
f_{c}=\frac{5.04}{\left(\frac{M}{L} \rho_{L}\right)^{1 / 2} z_{0}^{3}} \mathrm{M}_{\odot \mathrm{pc}^{-3}}\left(\mathrm{kms}^{-1}\right)^{-3} .
$$

where $\rho_{L}$ is the midplane luminosity density at the centre, and $z_{0}$ is the scale height. An $M / L$ of 7 is adopted, as derived in van der Kruit and Freeman (1984), and suggested in van der Kruit and Searle (1982). The comparison of the central values is reasonable on the basis that the most dense, most bound material likely sinks to the centre of the merging system (although this has not been established), and it is desirable that any outward decline of metallicity within a disk be preserved in the elliptical. 
Figure 1 shows the ellipticals as circles and hexagons (data taken from Lauer 1985 and Kormendy 1985,1986 ) and the disks as squares, with the Galaxy as an open square. The disk luminosities are calculated from the disk masses of van der Kruit and Searle 1982 using their M/L. On the basis of Figure 1, purely stellar mergers of bulgeless disk galaxies cannot make the cores of normal ellipticals. Mergers are allowed for the most luminous ellipticals, $M_{B} \lesssim-22$. Note that the density deficiency is two orders of magnitude for $M_{B}=-20$ ellipticals.

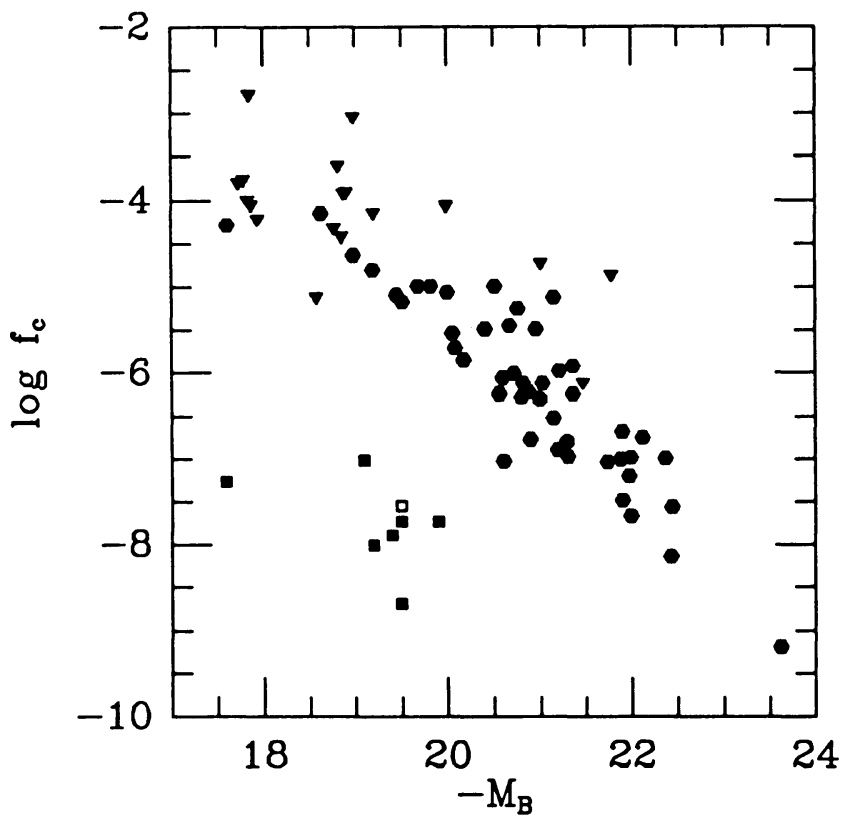

Figure 1. The central phase space densities of disks are shown as squares. Bulges and ellipticals are hexagons (Kormendy 1986) and circles (Lauer 1985). The inverted triangles are lower limits. $H_{0}=75$ for these measurements. Dissipationless merging can never move points upward.

A bulge in a disk galaxy completely overwhelms the phase space density of the disk. Bulges, being hot stellar systems, likely merge conserving their phase space density (Duncan, et al. 1983, Carlberg 1986). Since bulges appear to be part of the low luminosity extension of ellipticals in Figure 1, the merger of disks with bulges would move points horizontally forward placing them above the elliptical line in Figure 1. Candidates for old merged disk galaxies are the few normal ellipticals where the phase space densities are exceptionally high for their luminosity, possibly, those with only lower limits in Figure 1.

There are two conclusions that could be drawn from this comparison of disk galaxies and ellipticals. If mergers of disks make ellipticals, then the cores of ellipticals must be almost completely composed of stars subsequently added to the core, possibly through gas sinking to the centre and turning into stars. In this case, the cores could have different kinematics, for instance, more rotation; and possibly different metal abundances than the rest of the galaxy. The core buildup must be done in such a way that the fairly tight correlations between core radius, core surface brightness, and total luminosity arise (Kormendy 1985, Lauer 1985). A more extreme conclusion, and one that is premature in the absence of a better understanding of gas and star formation, is that mergers do not make most normal ellipticals. 


\section{GALAXY FORMATION IN A DISSIPATIVE COSMOLOGY}

Dissipative formation models for individual ellipticals have been examined by Larson (1975), Miller and Smith (1981) and Carlberg (1984a,b). These experiments fairly successfully reproduce the typical elliptical's density profile, the consequence of the very rapid star formation allowing a nearly dissipationless collapse of the stellar component. The experiments of Carlberg $(1984 \mathrm{a}, 1985)$ show that similar parameters for star formation and gas cooling can give rise to ellipticals, bulges, and disks depending on the ratio of core to envelope mass in the protogalaxy as it turns around from the Hubble flow.

The uncertainties in numerical experiments of galaxy formation are manifold. Simple gas physics can be adequately simulated, leaving the main uncertainties in the initial conditions. All the models reported above chose nearly uniform density and velocity fields as starting points and evolved the objects in isolation. Cosmological experiments show that the initial conditions are likely to be very nonuniform. Furthermore, since present day galaxies interact substantially with one another and their environment, at earlier times the protogalaxies probably did so even more.

To understand the relation of galaxies to large scale structure it is essential to have a basic understanding of the physics of a universe containing collisionless dark matter and gas. To this end, exploratory numerical experiments of the growth of galaxies within a two component universe are reported below. The gas physics and cosmological model are deliberately kept as simple as possible in order to highlight the general principles that are expected to hold for various initial conditions and more elaborate gas physics. The present analysis focuses on the growth of angular momentum in the two components.

\subsection{The Experiment}

Galaxy formation within a group or cluster of galaxies is an interesting situation, particularly since elliptical galaxies tend to be found in such high density neighbourhoods (Dressler 1980). The presence of considerable dark matter in the clusters is well established, and, in many, $\mathrm{X}$-ray data suggest that the dark matter is smoothly distributed (Jones et al. 1979). If the gas and dark matter were initially similarly distributed, then the current presence of galaxies as discrete units argues that they must shrink enough through dissipation to lower their collision cross-sections (White and Rees 1978).

The experiment starts with an isolated expanding sphere of $2 \times 10^{5}$ particles, randomly distributed. Since the particles are so numerous the amplitude of density fluctuations on large scales is small. The particle positions are further perturbed with an imposed white noise spectrum, added as several hundred randomly oriented waves. The velocities are left as pure Hubble flow, with an expansion velocity such that the initial $\Omega=1.28$. Half of the particles are collisionless and only interact via gravity, while the other half are subject to both gravity and collisions. One collisionless particle has a mass 10 times one gas particle. Initially the two particle distributions are identical. Gravity is evaluated on a grid 64 on a side, using an FFT method. The particles are advanced with a second order accurate method.

An isothermal gas is the simplest that loses energy under compression. Adopting this equation of state has the advantage of bypassing the complexities of cooling. The thermodynamic and transport properties of isothermal particles are fairly well defined, as opposed to, say, "sticky particles". Of course detailed realism has been sacrificed, but the aim of this exploratory study is to highlight the essential 
differences between a cooling gas and the collisionless background. The particles identified as gas are turned over to an algorithm that searches for nearest neighbours and tests them for approaching velocities. If there is a collision, then the particles are brought to their centre of mass and momentum, and assigned new relative velocities drawn from a Maxwellian distribution. This method is quick, has no problem with particle interpenetration, and is a fairly accurate reflection of the kinetics of gas atoms. Useful measurements require many particles to blur the particle microphysics simulation into an acceptable realization of the desired physical experiment. For these experiments there are usually 1000 or more particles per fragment, which is adequate for this investigation.

The problem of star formation is sidestepped here. These experiments are mainly investigating the properties of the protogalactic gas clouds that fragment out of the expansion. Adding star formation at this point would mostly confuse the issues. The fragments here are subject to considerable stirring from halo merging that would likely keep any stars that did form fairly well mixed with the gas. If the experiment was less overdense, $\Omega \leq 1$, some gas fragments would escape immediate inclusion in a cluster, and would ultimately dissipate down to flat, angular momentum supported, gas disks. To have any spheroids in such a situation it would be necessary to introduce star formation into the experiment.

The important physical length in this problem is the Jeans length,

$$
\lambda_{J}=\frac{2 \pi}{\sqrt{3}} R^{3 / 2} c_{s},
$$

in units where $G=M=R_{0}=1$, and where $R$ is the current size of the sphere, and $c_{s}$ is the sound speed of the isothermal gas. Experiments were done with $c_{s}$ of 0.01 and 0.03 giving 10 to 30 Jeans lengths across the experimental volume.

The particle viscosity is of considerable importance when angular momentum is an issue. In these experiments the effective Reynolds number of a fragment is,

$$
\Re=\frac{V_{0} \lambda_{J}^{2}}{\frac{1}{3} \ell c_{s}} .
$$

where $\ell$ is the mean interparticle seperation, and $V_{0}$ is the initial expansion velocity, 1.25 in the units here. The Reynolds number across a fragment is about 100 , which should be safe from viscous effects for the duration of these experiments, only a few dynamical times. As a check, collisions were turned off for particle separations less than 0.01 units, thereby stopping any gas viscosity in regions with overdensities at maximum expansion greater than about 1000 . No significant change was found in the results. It seems safe to conclude that angular momentum transfer in these experiments is dominated by gravitational torques.

\subsection{The Growth of Clustering in the Two Components}

Figure 2 shows an $\mathrm{x}-\mathrm{y}$ projection of the distribution of collisionless and gas particles at a time near maximum expansion. The collisionless particle distribution is essentially identical in both the cold and warm gas experiments, hardly surprising since the collisionless particles contain most of the mass. The gas particles have a strikingly different distribution, dependent on the temperature, they are much more compact and the number of gas fragments is strongly dependent on temperature. The small sizes of the gas fragments are of course due to dissipation, although their 
core sizes are largely determined by the softening of gravity (there are about 60 softening lengths across the pictures).

The collisionless particles cluster in the hierarchical manner appropriate to the prescribed fluctuation spectrum. Small mass fragments first appear and then merge together to form fewer larger groups. Eventually nearly all of the particles here will be incorporated into one large group. The gas particles cluster rather differently. Gas fragments do not appear until the size scale that is turning around exceeds the Jeans length. The fragment masses are rather broadly distributed around the Jeans mass. Following turnaround they collapse and dissipate on a dynamical time scale. The number of gas fragments decreases only a little with time, going from 24 to 18 over the time interval from 3.5 to 7.0 in a warm experiment. In contrast the number of collisionless particle fragments decreases from 71 to 33 over the same time interval. The shrinkage of the gas fragments is enough to "break the hierarchy", and reduce their merger rate below the undissipated rate (Aarseth and Fall 1980). Unfortunately the shrinkage in these two experiments is inadequate to allow the entire cluster of galaxies to survive the epoch of entire cluster collapse without considerable merging.

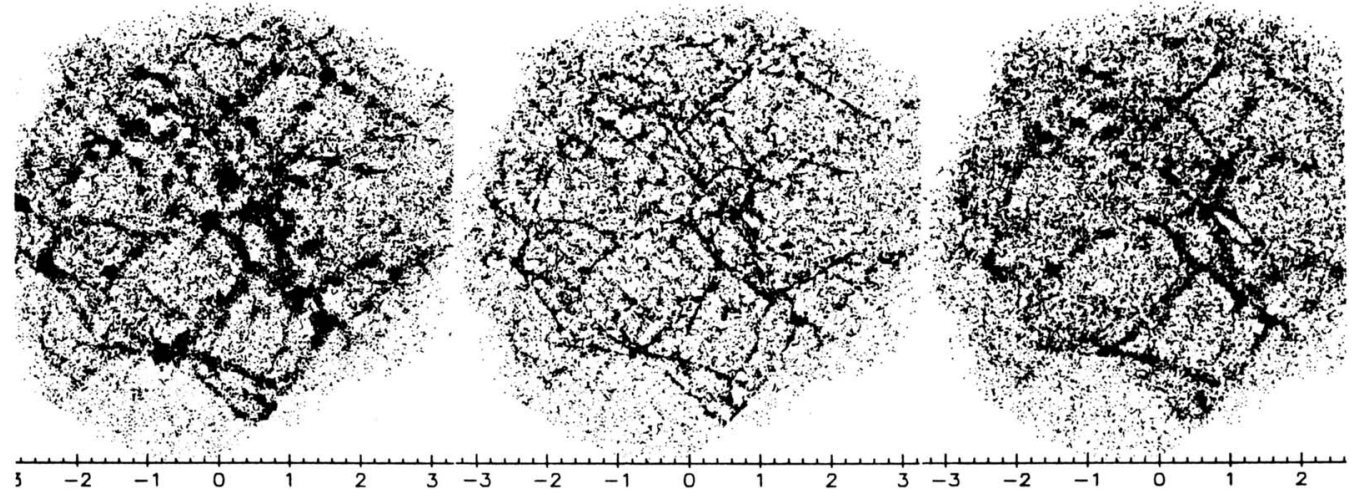

Figure 2. Projection on the $x-y$ plane of the particles at $t=4$. From left to right, collisionless particles, $c_{s}=0.01$ gas, and $c_{s}=0.03$ gas.

\subsection{Angular Momentum in the Fragments}

Angular momentum is acquired when the quadropole moment of the protogalaxy couples to the tidal field produced by neighbouring galaxies (Hoyle 1949). Elliptical galaxies have roughly a factor of six times less angular momentum than comparable mass disks (Fall 1983) so their spins are not compatible with being the tail of the general spin distribution. Some process is required to either pick out fragments that are likely to have low angular momentum (Blumenthal et al. 1984) or remove or dilute the angular momentum that is generated by tidal torques (Fall 1983, Frenk et al. 1985). The experiments reported here find an additional effect; the protogalactic gas has a disproportionately large amount of angular momentum at large radii where it is susceptible to stripping during the build up of clustering.

To identify groups for analysis, a "friends of friends" algorithm is used that joins all particles that are within a specified minimum distance of each other. The standard separation is 0.02 units, or about $1 / 5$ of the mean interparticle separation. The group radii and angular momenta are somewhat dependent on the choice of 
group density since the outer parts are constantly being disturbed, and the inner regions communicate with the outer parts via gravitational coupling. The chosen interparticle separation for inclusion in a group gives fragment properties that are well defined, and are repeatable at other seperations after suitable scaling.

To compare the properties of the collisionless matter with the gas a moment is chosen when the two types of fragments have nearly equal numbers of particles in them. Thus, in the warm $\left(c_{s}=0.03\right)$ experiment, the time 3.5, near maximum expansion, is chosen as the standard for analysis. Fragments are analyzed only if they contain at least 200 particles. About $80 \%$ of the collisionless particle fragments (halos) contain gas fragments (galaxies). In fact there is a close correspondence between the two types of particles, it just happens that some of them have insufficient numbers in their groups to be analyzed together.
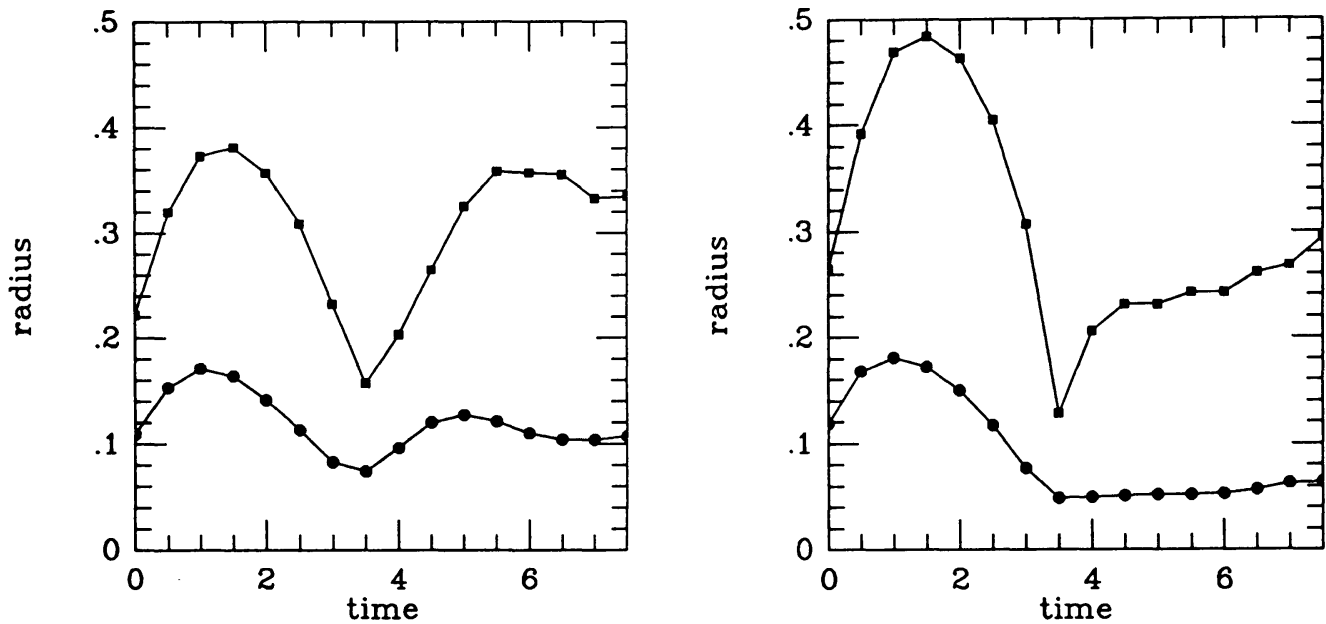

Figure 3. Average (circles) and maximum (squares) radii of groups in the collisionless (left) and gas (right) particles.

The positions of the particles that eventually come together to make the fragments identified at time 3.5 can be traced forward and backward in time. Figure 3 shows both the average radii and maximum radii of the fragments with time. The gas groups contain slightly more particles on the average so they are larger during the expansion phase. The separation of the gas fragments from the general clustering can be seen in Figure 3, since their average radius collapses to a well defined size, and stays there nearly to the end of the measurement time. The collisionless particles collapse, only to immediately re-expand as the original fragments are diluted by mergers with other groups. It is impressive that a little dissipative contraction (about a factor of 2 here) on top of a dissipationless collapse is sufficient to shut off a lot of merging in the gas component. The data given in $\S 3.2$ indicates the merging rate of the collisionless fragments is twice that of the gas fragments. 


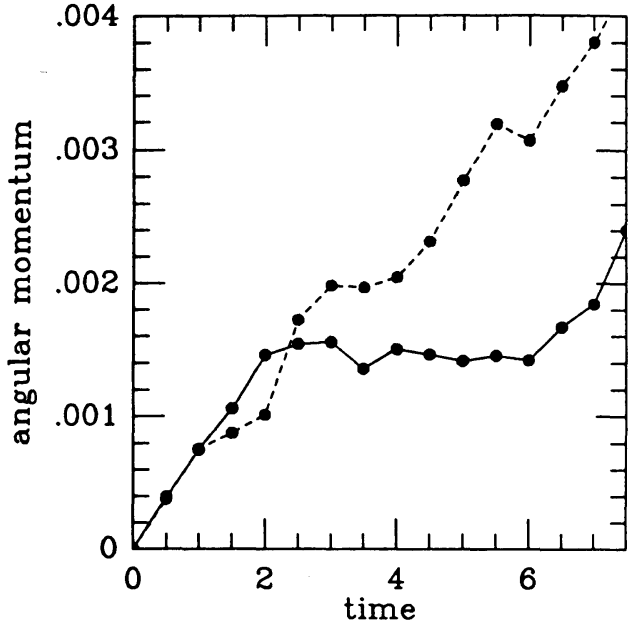

Figure 4. Average specific angular momentum vs time in the gas fragments (solid line) and collisionless fragments (dashed line) for particles in fragments identified at $t=3.5$.

The average specific angular momentum of the gas and collisionless particle fragments are displayed in Figure 4. The average specific angular momentum in the gas fragments is $70 \%$ of that in the collisionless fragments. The decoupling of the gas fragments from re-expansion and further spin up in larger units is again evident in Figure 4.

The spin of the gas is mostly acquired during the expansion phase, up to time 2.0. It is interesting to note that during the expansion phase both the gas and collisionless fragments gain angular momentum at the same rate, even though the gas groups are somewhat larger in size (see Figure 3 ) during this time. Since both components are experiencing the same tidal field the difference must be a result of different quadropole moments. Figure 5 shows the shape, measured as the geometric mean of the ratios of the two smaller principal moments of inertia to the largest one. The gas fragments stay significantly rounder during their expansion, leading to a smaller acquired angular momentum. The shapes are essentially the same after collapse. The difference in shape is not particularly large here, since most of the mass is in the collisionless component. If the gas is completely self-gravitating the effect is stronger. The relatively more spherical shape is a manifestation of the stabilization of the Lin-Mestel-Shu (1965) shape instability. A dynamical collapse of a constant density ellipsoid is stabilized against further flattening by a pressure of $3 / 5$ of the gravitational field on the short axis (Lynden-Bell 1964, 1979). Some help may also come from density gradient (see Figure 8) present in the fragments before collapse (Goodman and Binney 1983).

The most remarkable feature of the gas angular momentum is the relatively large fraction at large radii in the gas fragment. Figure 6 gives shows $M(h)$ curves, that is, the particles in a fragment are sorted by radius, then the cumulative mass is plotted against the cumulative angular momentum, both normalized to their maximum values. The $M(h)$ are shown at two times, the time of maximum expansion of the groups and at the time of group identification. Most of the gas angular momentum is at large radii. In fact, about $50 \%$ of the angular momentum lies in the outer $20 \%$ of the mass, and $80 \%$ lies in the outer $50 \%$ of the mass. At no time does the angular momentum distribution closely resemble that of a uniformly torqued constant density sphere, for reasons discussed with respect to Figure 8. 


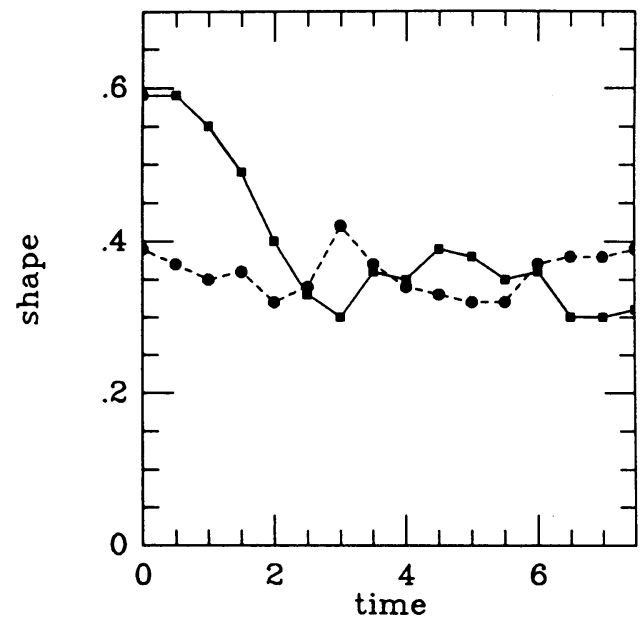

Figure 5. Shape vs time in the gas (solid line) and collisionless (dashed line) particle fragments. Perfectly spherical fragments would have a shape of unity. Most of the gas spin is acquired before time 2.0 .

The angular momentum transfer is a consequence of the gravitational coupling between the dissipationless material and the cooler, dissipating gas. The transfer of angular momentum is only very weakly dependent on the gas temperature, if the sound speed in the gas is lower than about $50 \%$ of the virial velocity within the fragment. If the gas sound speed is near the sound speed, or equivalently, if the gas is adiabatic then the gas and the collisionless particles have nearly identical angular momentum distributions.
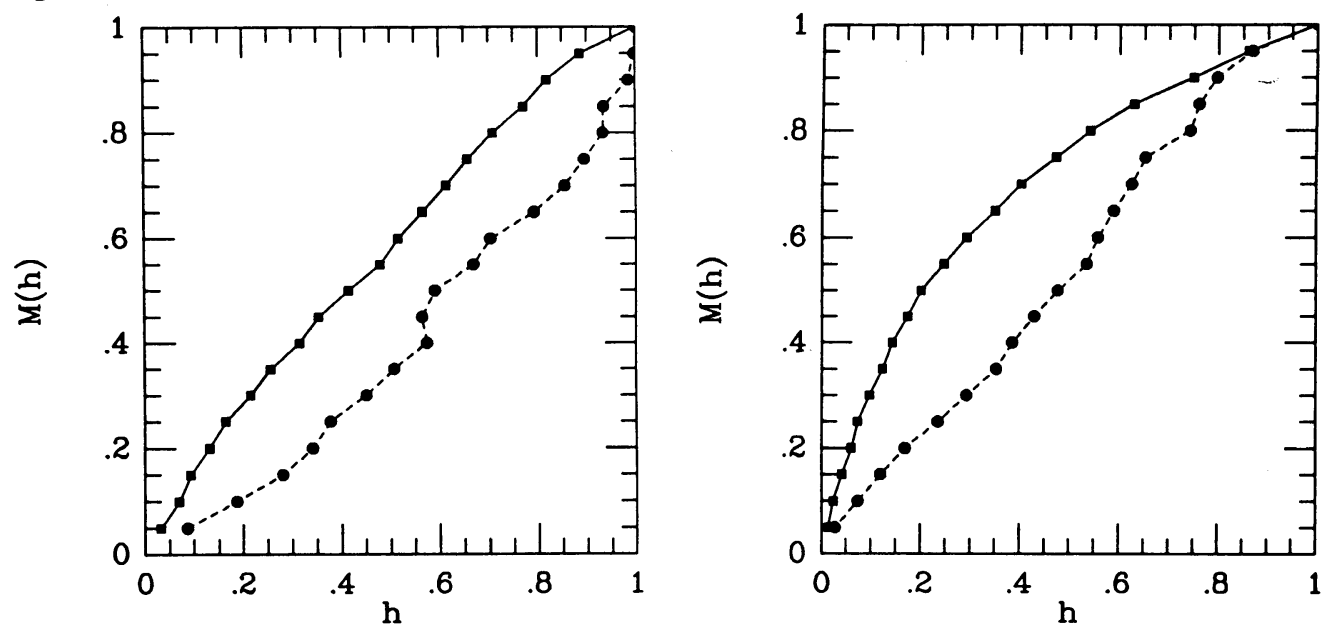

Figure 6. $M(h)$ distributions in the gas (solid line) and collisionless (dashed line) particles at time 1.5, maximum expansion, and time 3.5 , when the fragments were identified.

The average characteristic velocities of the gas particles are shown in Figure 7. The circular, radial infall, and circular streaming velocities are measured as spherical averages over shells. At the outermost radius of the gas its streaming velocity is 0.31 of the circular velocity, whereas the collisionless particles have a streaming velocity of only 0.18 at the same radius. This outermost gas can only 
collapse by a factor of 3 in radius before it is completely supported by angular momentum. A general collapse is also evident if Figure 7, but with a relatively low velocity At this rate it will take five dynamical times, as measured at the outside edge, for the material at the edge to fall in. This outermost gas must be allowed to settle in a quiet environment if it is to make it into a disk.

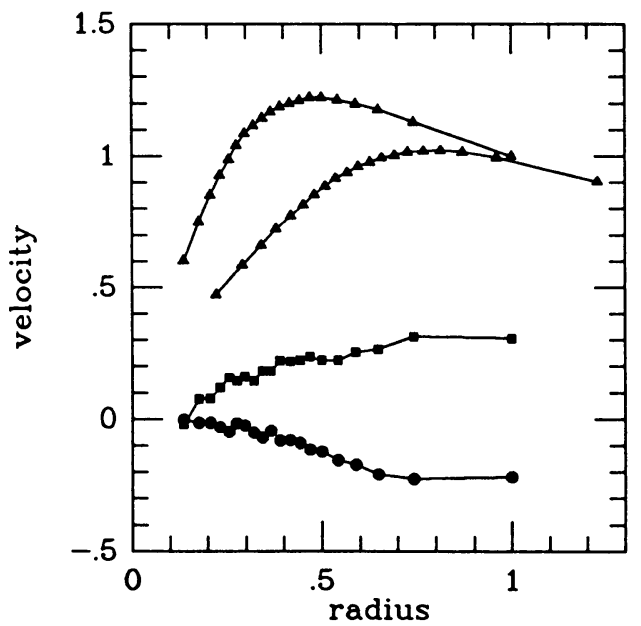

Figure 7. Characteristic velocities measured at time 3.5 normalized to the circular velocities at the edge of the fragments. Circles give the average radial velocity and squares the average azimuthal streaming velocity in the gas fragments. The upper line with triangles shows the circular velocity, $\sqrt{(G M / R)}$ assuming the gas is completely self-gravitating. The lower line with triangles shows the true normalized circular velocity from the collisionless particle mass distribution.

The objects that are collapsing are not cold, constant density spheres. Rather, the density declines outwards from the centre, so that the collapse time outside the core increases with radius. The radial density profile of the fragments at an early time and at the time of "turnaround" is shown in Figure 8 . The points are at $5 \%$ intervals of the total mass.

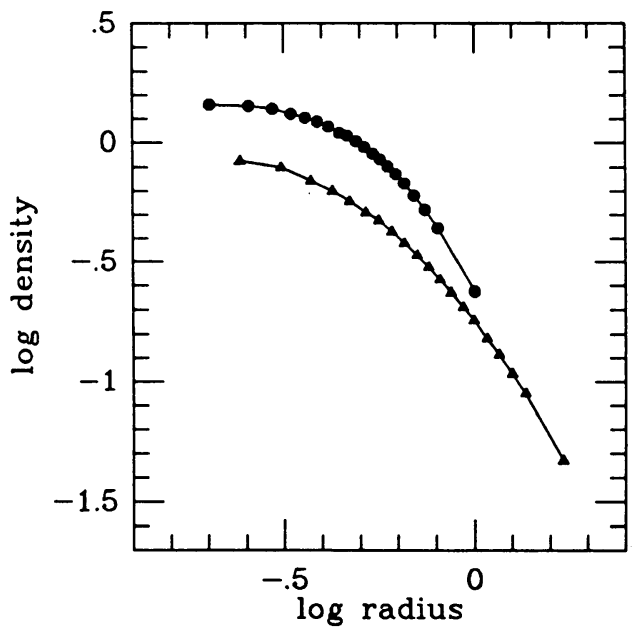

Figure 8. Average density profiles of the particles in the gas fragments identified at $t=3.5$, measured at the earlier times $t=$ 0 , the beginning, and $t=1.5$, turnaround.

During the collapse of the entire system, the gas fragments more or less all merge together to form a "hypergalaxy". Softening limits the collapse available to the fragments, that would core collapse leaving an envelope with $\rho \propto r^{-2}$ behind (Larson 1969). It is interesting to ask what might have happened had the cores been able to shrink more. Larson, Tinsley, and Caldwell (1980) provide a likely suggestion. The extended dark halos must come off the gas fragments to avoid too 
much mass segregation (White 1976), and metal enriched gas must come out of the galaxies to fill the cluster. Removal of half the gas mass would take $80 \%$ of the angular momentum. Furthermore the objects left behind have been subject to a great deal of violent relaxation from ongoing halo merging, making it difficult to form a very thin disk. It is tempting to identify these putative stripped gas fragments with bulges and ellipticals. A consequence would be that the Hubble type of an object would not be determined until the time of clustering of groups of galaxies.

\section{CONCLUSIONS}

The formation of ellipticals is likely to be strongly dependent on environmental influences, such as the clustering environment and interactions with other galaxies. Ellipticals are dynamically distinct as having relatively low angular momentum content, and high random velocities. The spin of a galaxy is generated by tidal torques, reduced by stripping, and diluted by merging. These three mechanisms have been investigated for their relation to dissipation. Mergers of present day disk galaxies would not make normal elliptical galaxies in the absence of some dissipative star formation to build up the core density. However the most luminous ellipticals have sufficiently large cores that there is no objection to mergers. For normal ellipticals it is not known whether gas resident in a disk could be brought to the centre and turned into stars to build up the core density to the observed values. The angular momentum imparted to a protogalaxy by tidal torques is slightly reduced in the presence of gas pressure, by about a one third. The reduction is due to the decreased quadropole moment as a result of partial pressure support of the protogalaxy and suppression of the Lin-Mestel-Shu instability. The main effect of dissipation is to allow gravitational torques associated with violent relaxation to transport the bulk of the angular momentum in the protogalaxy to large radii, where it could be stripped off during the growth of clustering in the collisionless component.

\section{REFERENCES}

Aarseth, S. J. and Fall, S. M. 1980, Ap. J., 236, 43.

Binney, J. J. 1977, Ap. J., 215, 483.

Blumenthal, G. R., Faber, S. M., Primack, J., and Rees, M. J. 1984, Nature, 311, 517.

Carlberg, R. G. 1984a, Ap. J., 286, 403.

Carlberg, R. G. 1984b, Ap. J., 286, 416.

Carlberg, R. G. 1985, in The Milky Way Galaxy, I.A.U. Symp. No. 106, ed. H. van Woerden, p. 615.

Carlberg, R. G. 1986, Ap. J., 309, in press.

Davies, R. L., Efstathiou, G., Fall, S. M., Illingworth, G., and Schechter, P. L. 1983, Ap. J., 266, 41.

Dressler, A. 1980, Ap. J., 236, 351.

Duncan, M. J., Farouki, R. T., and Shapiro, S. L. 1983, Ap. J., 271, 22.

Efstathiou, G., Fall, S. M., and Hogan, C. 1979, M. N. R. A. S., 189, 203.

Fall, S. M. 1983, in Internal Kinematics and Dynamics of Galaxies, I.A.U. Symp. 100, ed. E. Athanassoula, (Reidel: Dordrecht). 
Fish, R. A. 1964, Ap. J., 139, 284.

Frenk, C., White, S. D. M., Efstathiou, G., and Davis, M. 1985, Nature, 317, 670. Goodman, J. and Binney, J. 1983, M. N. R. A. S., 203, 265.

Hoyle, F. 1949, in Problems in Cosmical Aerodynamics (Central Air Documents Office: Dayton, Ohio), p. 195.

Illingworth, G. 1977, Ap. J. (Letters), 218, L43.

Jones, C., Mandel, E., Schwarz, J., Forman, W., Murray, S. S., and Harnden, F. R., Jr. 1979, Ap. J. (Letters), 234, L21.

Kormendy, J. 1985, Ap. J., 295, 73.

Kormendy, J. 1986, private communication.

Larson, R. B. 1969, M. N. R. A. S., 145, 271.

Larson, R. B. 1975, M. N. R. A. S., 173, 671.

Larson, R. B., Tinsley, B., and Caldwell, N. 1980, Ap. J., 237, 693.

Lauer, T. R. 1985, Ap. J., 292, 104.

Lin, C. C., Mestel, and Shu, F. H. 1965, Ap. J., 142, 1431.

Lynden-Bell, D. 1964, Ap. J., 139, 1195.

Lynden-Bell, D. 1979, Observatory, 99, 89.

Miller, R. H. and Smith. B. F. 1981, Ap. J., 244, 467.

Toomre, A. 1977, in Evolution of Galaxies and Stellar Populations, ed. B. M. Tinsley and R. B. Larson, (Yale Observatory: New Haven).

Rees, M. J. and Ostriker, J. P. 1977, M. N. R. A. S., 179, 451.

Silk, J. 1977, Ap. J., 211, 638.

van der Kruit, P. C. and Freeman, K. C. 1984, Ap. J., $278,81$.

van der Kruit, P. C. and Searle, L. 1982, Astr. Ap., 110, 61.

White, S. D. M. 1976, M. N. R. A. S., 174, 19.

White, S. D. M. and Rees, M. J. 1978, M. N. R. A. S., 183, 341.

\section{DISCUSSION}

Lake: In using phase-space densities to constrain merging, you compare central phase densities. Shouldn't you use peak phase densities?

Carlberg: The peak phase-space density in an elliptical is in the core. In a disk the phase-space density increases away from the centre, peaking someplace in the outer disk. At the present time it is completely unclear what disk material will actually end up at the centre of the merged system. The higher phase-space density outer parts of a disk are very cool and likely very susceptible to phase-space dilution. If the disks were test particles imbedded in halos, we know that the central halo particles of the merged system come largely from the centres of the precursor objects. In any case, the peak phase-space density is only a factor of 10 larger than the central phase-space density, so the argument is not greatly affected.

White: Could you clarify your conclusion about the viability of forming ellipticals from "real" spirals which almost always have bulges whose phase-space density is inferred to exceed that required in the elliptical remnant?

Carlberg: Bulges are viewed as the low luminosity extension of the spheroidal sequence. As shown in Figure 8 the protogalaxy has a core of nearly constant density that likely collapses with considerable violent relaxation. The size of the 
core in a particular protogalaxy would then determine the size of the spheroid. This is clearly extremely speculative, and needs further investigation with higher resolution experiments.

Gerhard: If I wanted to merge disk galaxies to make ellipticals, I would try to do this at early times. Two ways then come to mind by which your phase-space constraint could be circumvented. (i) At early times, the disks contain more gas, which would in a merging event go to the center and increase the central phasespace density of the forming elliptical. (ii) The diffusion process increasing the velosities in disk stars, as discussed by Wielen (1977, Astr. Astrophys., 60, 263), would not have had time to operate. To show how sensitive the phase-space density of disk material is to this process, let me make the extreme assumption that all stars had only one third of their present peculiar velocity dispersion, i.e., $10 \mathrm{~km} / \mathrm{s}$ instead of $30 \mathrm{~km} / \mathrm{s}$ in the solar neighborhood. Then the disk $z$-scalelength is reduced by a factor of 10 , and the phase-space density increased by a factor of 100 , according to your formula.

Carlberg: Certainly disks would have had higher gas contents and phase-space densities at earlier times. Mergers of very young gas disks are of course completely dominated by dissipative processes. The phase-space densities of the stellar component for a young disk can be estimated by imposing the constraint that the disk have a $Q$-value between 1 and 2 . At constant $Q$, the phase-space density increases as the inverse cube of the surface density. Two or three orders of magnitude increase in phase-space density would require a surface density down by about a factor of ten. That means that the disks must be very young, implying a similarly reduced stellar mass and the presence of a great deal of gas in the vicinity.

Kochhar: Why do ellipticals have to come from a merger of spirals?

Carlberg: Making ellipticals from spirals has a number of attractive features (Toomre 1977, Ann. Rev. Astr. Astroph., 15, 437). In particular, merging avoids having two very different sites for star formation: very rapid star formation in a collapsing spheroid, and slow formation in disks. Mergers are of course seen to occur, and it is hypothesized that the merged systems resemble ellipticals.

\section{Djorgovski: Is M32 a merger of two spirals?}

Carlberg: M32 has such an enormous central phase-space density (around 0.1 units) that it doesn't even fit on Figure 1. There are no obvious precursors that have the appropriate properties to make M32.

White: In your talk you didn't mention dissipative models for elliptical formation which are analogous to observed cooling flows. In such systems stars may have formed during subsonic settling of gas in a temporarily static potential well. There has been done recent work in this area stimulated by observations of cooling flows. Would you or someone in the audience like to comment on it?

Carlberg: To some degree, all dissipative models for galaxy formation resemble cooling flows. It is a matter of knowing the Jeans mass of the cooling region. The extreme possibilities are that the entire galaxy is a hot gas of one Jeans mass that 
is in nearly hydrostatic equilibrium and cooling on a free fall time scale only in its central regions, as in a cooling flow. The other possibility is that the galaxy is made up of many Jeans masses, each of which is a little cooling flow. The time scales in the many small subgalactic clumps may be sufficiently short that most of the star formation would occur in them, and subsequently merge together to form the entire galaxy. Of course, the cooling time argument suggests that galaxies sit right near the dividing line of the two regimes.

Richstone: I was impressed that violent relaxation always gives you $\sigma_{r}>\sigma_{z}$ at large $r$. Can you make a similar statement about models where dissipation is important?

Carlberg: The velocity dispersion in the gas is isotropic, so that it is unlikely that the velocity ellipsoid can ever have a larger tangential component than radial component. In fact, in the models of Carlberg (1984a) it was found that the kinematics of dissipative models were generally similar to those of violently relaxed dissipationless models.

Schechter: The two key processes in your prescription for galaxy formation are merging and stripping. The merging occurs naturally in your simulations but the stripping is done in an ad hoc manner. Is there any prospect of a more realistic treatment of stripping?

Carlberg: The prospects for a more realistic treatment of stripping are excellent, provided that computer time continues to be available in abundance. It is mostly a matter of improving the gas physics. Based on the experience gained here improved methods are currently under development.

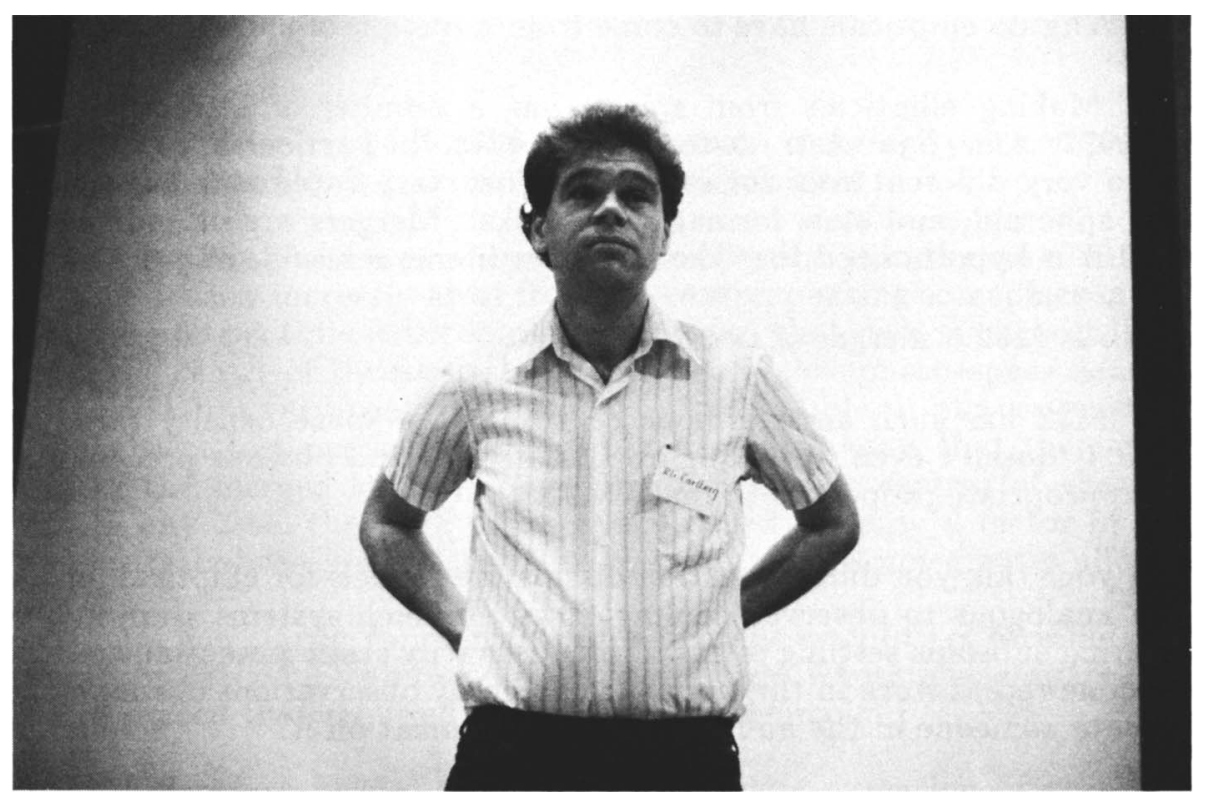

Ray Carlberg explains galaxy formation. 